\title{
Desarrollo de un modelo geológico 3D en el centro urbano de Oviedo (Asturias)
}

\author{
Pelayo Fernández-Álvarez y Luis Pando \\ Departamento de Geología de la Universidad de Oviedo, C/ Jesús Arias de Velasco s/n, 33005, Oviedo, Asturias. \\ U0224440@uniovi.es y Ipando@geol.uniovi.es
}

\begin{abstract}
RESUMEN
En relación a las investigaciones sobre geología urbana en Asturias (NO de España) se presenta el primer modelo tridimensional basado en capas acerca de la configuración geológica del centro de Oviedo. El modelo obtenido está fundamentado en los datos sobre el subsuelo proporcionados por casi un millar de puntos de reconocimiento georreferenciados, incluyendo más de 500 sondeos mecánicos, cubriendo un área de aproximadamente $3 \mathrm{~km}^{2}$. Esta información, almacenada en una base de datos geotécnicos, fue gestionada empleando un Sistema de Información Geográfica. Tras preparar un Modelo Digital de Elevaciones a modo de referencia altitudinal, han sido generadas diferentes superficies ráster por medio de un interpolador determinístico y álgebra de mapas, representando los contactos entre las unidades geológicas. Esto ha permitido revisar y actualizar la cartografía geológica superficial; investigar la geometría de las unidades, principalmente sus espesores; y deducir el trazado de potenciales fallas que cruzan el subsuelo urbano. Todo ello ha sido integrado en un entorno virtual 3D complementado en superficie mediante la recreación de diversos elementos del entramado urbano.
\end{abstract}

Palabras clave: 3D, geología urbana, interpolación, modelo, SIG.

\section{Development of a 3D geological model in the urban center of Oviedo (Asturias)}

\begin{abstract}
In relation to the research on urban geology in Asturias (NW Spain), this is the first layer-based three-dimensional model about the geological setting of Oviedo's urban centre. Its development is based on data on the subsoil provided by almost a thousand geo-referenced site investigation points, including more than 500 boreholes, covering an area of approximately $3 \mathrm{~km}^{2}$. These data, stored in a geotechnical database, were managed through a Geographical Information System. After producing a Digital Elevation Model as an altitudinal reference, different raster surfaces were generated by using a deterministic interpolator and map algebra, representing the boundaries between the geological units. This allowed the reviewing and updating of the geological superficial mapping; investigating the geometry of the units, mainly their thicknesses, and deducting the traces of potential faults crossing the urban subsurface. All this has been integrated into a virtual 3D environment complemented through the recreation of different urban elements.
\end{abstract}

Keywords: 3D, GIS, interpolation, model, urban geology.

\section{Introducción}

El extensivo uso de reconstrucciones virtuales 3D del medio geológico, permite amplias y crecientes posibilidades en el tratamiento e interpretación de datos del terreno en variadas escalas de trabajo. Estas reconstrucciones están basadas, por lo general, en cartografías geológicas, observaciones en afloramientos, sondeos de reconocimiento, mallas de cor- tes geológicos y secciones geofísicas, entre otros datos. La provisión por tanto de datos en cantidad y calidad es indispensable para nutrir a los modelos, por lo que en numerosos casos su expansión ha transcurrido en paralelo a la relevancia que han adquirido las bases de datos geológicos y geotécnicos digitales (Veeger et al., 2004).

La metodología de modelización basada en capas es muy común y comprende la creación de un con- 
junto de superficies que representan variaciones en la geología (p. ej., litológicas), obtenidas mediante interpolaciones. La utilización de técnicas geoestadísticas implica complejidad operativa, pero permite generar modelos más avanzados y fácilmente actualizables. Hoy día existe una tendencia cada vez mayor a componer virtualizaciones que convierten el subsuelo en una matriz de unidades cúbicas. Esto permite dotar de volumetría al terreno posibilitando que a cada elemento -vóxel- se le pueda asignar propiedades y analizar sus variaciones espaciales en el entorno 3D (p. ej., Tonini et al., 2009, Stafleu et al., 2011, Hoyer et al., 2015).

En el ámbito urbano, el desarrollo de bases de datos y la modelización del subsuelo han despertado gran interés durante la última década por su aplicabilidad al ordenamiento espacial de las ciudades (Royse et al., 2015). La integración del espacio subterráneo en el planeamiento territorial da lugar a colaboraciones como la Acción COST Sub-Urban, red europea que involucra administraciones locales, servicios geológicos y otros centros de investigación (Campbell et al., 2014). Así, son ya diversas las actuaciones encaminadas a estudiar subsuelos urbanos mediante modelizaciones, sobre todo en capitales y zonas metropolitanas de cierta entidad como Londres, Viena, Oslo, Glasgow, Dublín, Rotterdam, Bruselas, Helsinki o Belfast. No obstante, estas investigaciones se están extendiendo a más poblaciones y más pequeñas (Mielby and Sandersen, 2017); en cada contexto, como es lógico, con la sistemática de trabajo adaptada a las características específicas del sustrato geológico y a las necesidades generadas por la política de desarrollo urbano. Con el denominador común del análisis geométrico de las unidades geológicas, otros de los propósitos más frecuentes son la identificación de riesgos (Merritt et al., 2009), atribución de propiedades a los materiales (Royse et al., 2009), gestión del agua subterránea (Campbell et al., 2010) o la protección del patrimonio arqueológico (De Beer et al., 2012). A ello se suma el potencial que ofrece la visualización combinada con la representación 3D de edificaciones, viario y otras infraestructuras (Eriksson et al., 2016). Recrear el entorno supone una herramienta de comunicación de resultados muy valiosa de cara a los destinatarios de los modelos, en buena medida técnicos implicados en la planificación territorial.

En el caso de Asturias, las investigaciones sobre geología urbana se han centrado en el estudio de las principales ciudades, las cuales encabezan un área metropolitana poblada por unos 800.000 habitantes. Oviedo y Gijón cuentan con sendos monográficos dedicados a sus subsuelos (Gutiérrez Claverol and Torres Alonso, 1995, Gutiérrez Claverol et al., 2002) entre otras contribuciones. Para ambas ciudades, así como para Avilés, se han desarrollado bases de datos geológicos y geotécnicos implementadas en Sistemas de Información Geográfica (SIG) que integran, entre otra, información de millares de prospecciones (Pando et al., 2014, Díaz-Díaz, 2016, Pando et al., 2016). Dichos repositorios son objeto de provecho científico estando abiertas en la actualidad diversas líneas de trabajo, entre ellas la modelización 3D del espacio subterráneo (Pando et al., 2018). En relación a ello la investigación que se presenta dispuso para Oviedo los siguientes objetivos: i) actualización de la cartografía geológica superficial del centro urbano; ii) estudio de la geometría en profundidad de las unidades geológicas que conforman las primeras decenas de metros, ámbito en el que se realizan la gran mayoría de excavaciones antrópicas; iii) identificación de posibles fallas de escala cartografiable; iv) integración de la información geológica generada en un entorno tridimensional.

\section{Zona de estudio}

Oviedo posee una población de 215.000 habitantes y está situada sobre una depresión alineada en dirección NE-SO, con altitudes entre 160 y $330 \mathrm{msnm}$, y $5^{\circ}$ de pendiente promedio. El área de estudio, rectangular en planta y con extensión de 3,2 $\mathrm{km}^{2}$ (Fig. 1a), comprende lo que tradicionalmente se ha considerado el centro de la ciudad. Integra el casco histórico y está delimitado por el anillo que configuran las principales vías de comunicación interna del entramado urbano (Fig. 1b).

Geológicamente la zona se localiza en el extremo SO de una depresión de $80 \mathrm{~km}$ de longitud denominada "depresión prelitoral de Oviedo-Cangas de Onís" (García-Ramos and Gutiérrez Claverol, 1995) o "cuenca meso-terciaria de Oviedo" (Alonso et al., 1996). Dentro de ésta, la ciudad se asienta sobre materiales cretácicos y cenozoicos discordantes sobre formaciones paleozoicas (Fig. 1c). Estas últimas, con altos buzamientos, conforman un zócalo muy tectonizado (Fig. 1d) perteneciente a la Zona Cantábrica, sector más externo y septentrional del Macizo Hespérico (Julivert et al., 1972).

La estratigrafía de esta cuenca está formada por unidades cretácicas que alternan naturaleza silícea y carbonatada (González Fernández et al., 2004). En el área urbana están representadas las tres formaciones del Cretácico Superior más altas de la serie, que de muro a techo son:

- Fm. San Lázaro. Unidad con predominio de calizas nodulosas (Fig. 2a), puntualmente muy 

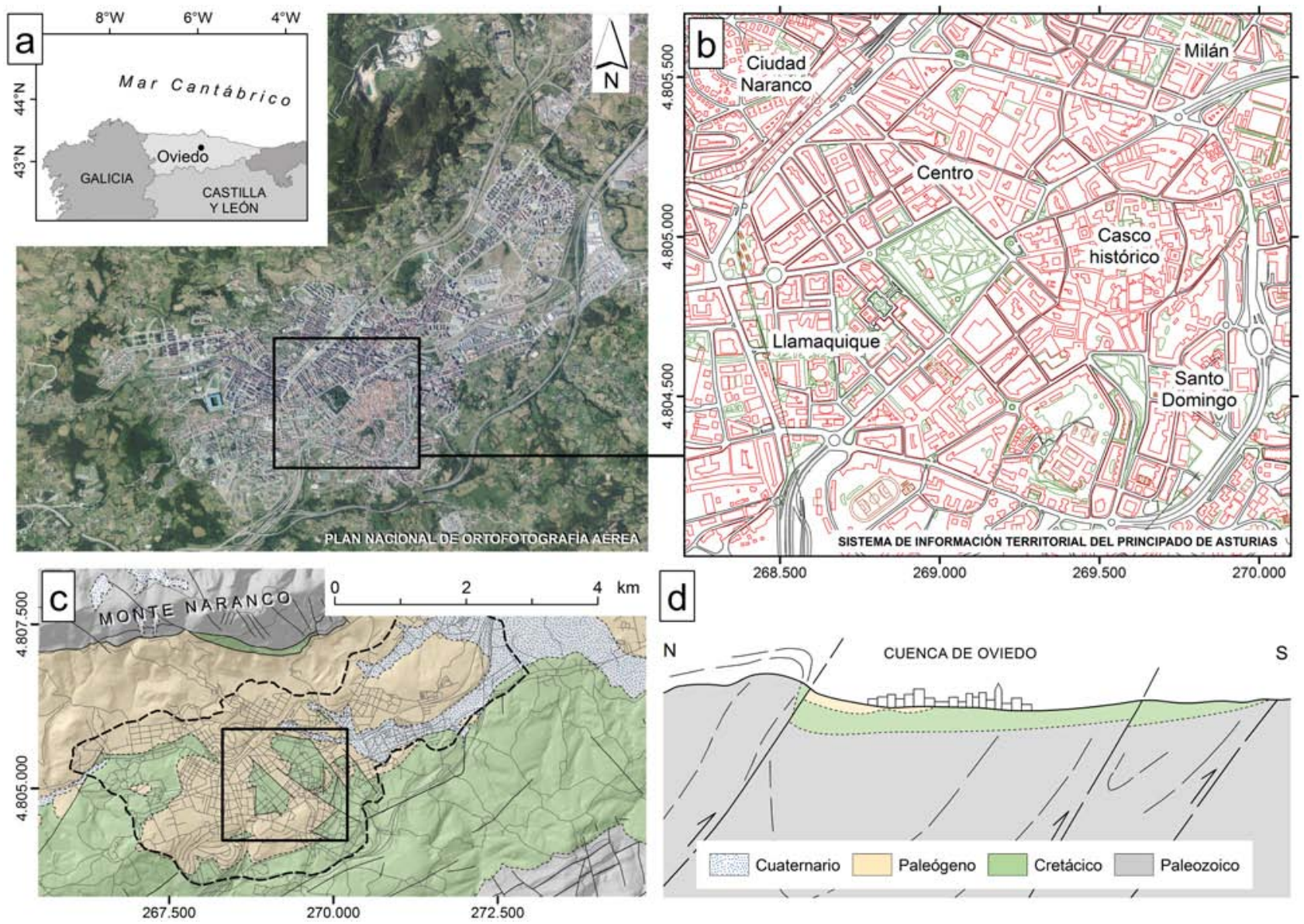

Figura 1. (a) y (b) Contexto geográfico de la zona de estudio; (c) Mapa geológico de síntesis elaborado con datos propios; (d) Sección esquemática N-S de la cuenca (modificada de Pulgar et al., 1999).

Figure 1. (a) and (b) Geographic context of the study area; (c) Simplified geological map elaborated with own data; (d) N-S schematic cross section of the basin (modified from Pulgar et al., 1999).

karstificadas, con tramos de lutitas. Hacia techo pasa a limos y areniscas en tránsito a la formación superior. Parte de estas rocas fueron aprovechadas para construcción en el casco histórico. AI SE de la ciudad se tiene constancia de un espesor de $48 \mathrm{~m}$ (Pando et al., 2014).

- Fm. La Argañosa. Es de naturaleza siliciclástica y está compuesta por arenas finas a muy gruesas, con cantos dispersos, e intercalaciones de gravas, conglomerados y microconglomerados cuarcíticos, así como intercalaciones de limos y arcillas (Fig. 2b). Su granulometría, con frecuentes horizontes permeables y piezometrías muy someras, determina un importante sistema acuífero multicapa (González Fernández et al., 2005). Asimismo, ha sido explotada en areneros y arcilleras. Muestra en la cuenca un espesor de entre 30 y $40 \mathrm{~m}$.
- Fm. Oviedo. Comprende hacia muro calizas bioclásticas, arenosas y nodulosas de grano fino. Hacia techo predominan las calizas arenosas y areniscas calcáreas con tramos brechoides (Fig. 2c). Estas litologías fueron antaño extraídas para la construcción de monumentos. Localmente se reconocen karstificaciones -cavidades y dolinas- así como una intensa arenización hacia el sector oriental con hasta 7 $\mathrm{m}$ de depósito eluvial (Gutiérrez Claverol et al., 2008). La formación rebasa los $40 \mathrm{~m}$ de espesor al E de la cuenca, pero llega a desaparecer cuando ha sido localmente erosionada por completo antes del depósito de los materiales cenozoicos.

Sobre los materiales descritos yace discordante mediante paleorrelieve una cobertera paleógena fluvio-lacustre (Fig. 2d). Se diferencian en ella tres gran- 

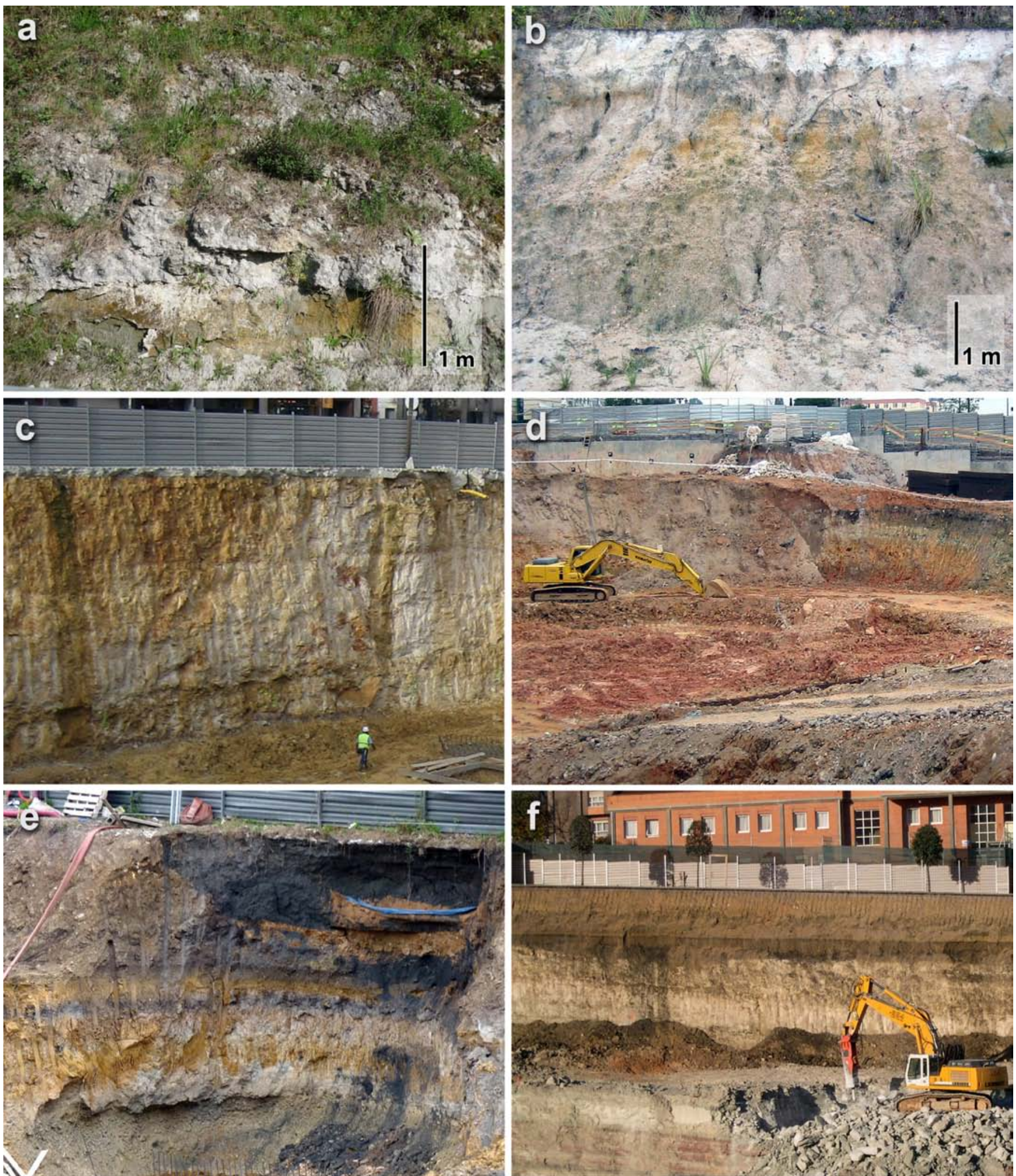

Figura 2. Aspecto de las principales unidades que forman el subsuelo urbano de Oviedo: (a) Fm. San Lázaro; (b) Fm. La Argañosa; (c) Fm. Oviedo; (d) Paleógeno; (e) Sedimentos aluviales; (f) Rellenos antrópicos.

Figure 2. Appearance of the different units which form the Oviedo urban subsoil: (a) San Lázaro Formation; (b) La Argañosa Formation; (c) Oviedo Formation; (d) Paleogene; (e) alluvial sediments; (f) man-made deposits. 
Pelayo Fernández-Álvarez y Luis Pando, 2019. Desarrollo de un modelo geológico 3D... Boletín Geológico y Minero, 130 (3): $489-503$

des conjuntos litológicos, con notables cambios laterales de facies, cuyo espesor conjunto supera los 200 m (Gutiérrez Claverol and Torres Alonso, 1995). A grandes rasgos el tramo inferior es el más carbonatado, con margas en alternancia con calizas y dolomías blancas -beneficiadas en su momento como piedra ornamental-. Este tramo presenta a veces naturaleza yesífera, en algunas zonas como capas métricas masivas de yeso con evidencias de karstificación de cierta entidad (González Fernández et al., 2010; Pando et al., 2013). En el tramo medio abundan las arcillas, margas arcillosas y margas versicolores con niveles ocasionales de caliza y cristales de yeso. El tramo superior, más siliciclástico, está compuesto por areniscas calcáreas, calizas, arenas y margas con horizontes de gravas, y conglomerados silíceos.

En relación a los sedimentos cuaternarios, los depósitos fluviales más extensos, con una potencia media de 4-6 $m$, configuran una planicie aluvial en el margen NE (Fig. 2e). También se encuentran sedimentos de pequeños arroyos ya desaparecidos, de mucha menor entidad y ocultos bajo el entramado urbanístico. Por otro lado, tapizando la ladera meridional del monte Naranco, se han acumulado sobre el sustrato paleozoico y la cobertera paleógena coluviones con espesores superiores a $15 \mathrm{~m}$. Finalmente, los rellenos antrópicos presentan diverso origen y naturaleza (Fig. 2f). Corresponden a explanaciones de urbanización; vertidos para saneamiento de terrenos; obras de tierra levantadas por las obras civiles; y escombreras y rellenos de antiguas canteras, areneros y arcilleras. Su espesor fluctúa si bien suele mantenerse por debajo de $2 \mathrm{~m}$.

En términos estructurales la cuenca meso-terciaria muestra rasgos vinculados a la reactivación de estructuras variscas relacionada con la fase compresiva alpina que provocó en Asturias un acortamiento $\mathrm{N}-\mathrm{S}$, responsable de la deformación de las cuencas mesozoicas y del levantamiento de la Cordillera Cantábrica (Alonso et al., 1996; Pulgar et al., 1999). Esta deformación ha conformado un sinclinal asimétrico de trazado aproximado E-O. Su flanco meridional, donde se sitúa mayoritariamente la ciudad de Oviedo, se inclina por debajo de los $20^{\circ}$ hacia el N, mientras que el septentrional adopta una disposición más verticalizada (Fig. 1d). La discordancia que separa la serie cretácica del Paleógeno muestra un bajo ángulo; las litologías cenozoicas, menos basculadas que las mesozoicas, presentan buzamientos con frecuencia inferiores a $10^{\circ}$. Las fallas que afectan a la cuenca son en su mayoría subverticales y han sido cartografiadas en el entorno urbano, reconociéndose tres orientaciones preferentes (Gutiérrez Claverol and Torres Alonso, 1995): NE-SO, NO-SE a NNO-SSE, y E-
O. En ocasiones también se identifican pequeñas fallas de alto ángulo y naturaleza inversa.

\section{Metodología}

Ha sido adaptada a las necesidades planteadas, las particularidades del subsuelo ovetense y los recursos disponibles (Fig. 3). Como herramienta informática se utilizó un Sistema de Información Geográfica, el software ArcGIS (Esri), plataforma empleada en el desarrollo de otros modelos 3D suburbanos basados en capas (p.ej., Devleeschouwer and Pouriel, 2009). La elección está fundamentada en que un SIG encaja con la procedencia de los datos usados sin necesidad de adecuar su estructura a los formatos de entrada que precisan otras aplicaciones; permite amplias capacidades de consulta, tratamiento y análisis espacial; y posibilita una integración visual muy efectiva.

En la primera fase de trabajo se preparó el marco geográfico y un Modelo Digital de Elevaciones (MDE), para localizar espacialmente las prospecciones, y a partir de ello todos los productos derivados. Se acudió a la cartografía topográfica autonómica 1:5.000 más reciente (2003), difundida por el SITPA-IDEAS (Sistema de Información Territorial del Principado de Asturias y la Infraestructura de Datos Espaciales) en archivos CAD. Puntualmente fue necesario actualizar el parcelario y construcciones previa consulta catastral a través de acceso remoto mediante WMS (Web Map Service), con el apoyo de la ortoimagen más actual del Plan Nacional del Ortofotografía Aérea. Para disponer de un MDE óptimo se valoraron dos alternativas: generarlo a partir de la cartografía autonómica citada, o crear uno con los datos LIDAR (Laser Imaging Detection and Ranging) proporcionados por el Instituto Geográfico Nacional y fechados para la zona de estudio en 2012.

En cuanto a la documentación geológica se recabaron dos cartografías: la publicada por Gutiérrez Claverol and Torres Alonso (1995) -que fue georreferenciada- por su escala (1:14.000), y la facilitada por el IGME a través del Plan GEODE por su actualidad. La información subterránea la proporcionó la base de datos geológicos y geotécnicos de Oviedo (Pando et al., 2014). Se tomaron los registros de 507 sondeos mecánicos, con una profundidad promedio de $14,5 \mathrm{~m}$ y máximo de $67 \mathrm{~m}$, representando en total 7.352 metros de terreno. Estas prospecciones aportan cota de boca, y tramificación de las unidades geológicas seccionadas y sus espesores. Asimismo fueron incorporadas 447 observaciones in situ provenientes de zanjas, vaciados de solares, obras de cimentación y otros reconocimientos. Representa en conjunto más 


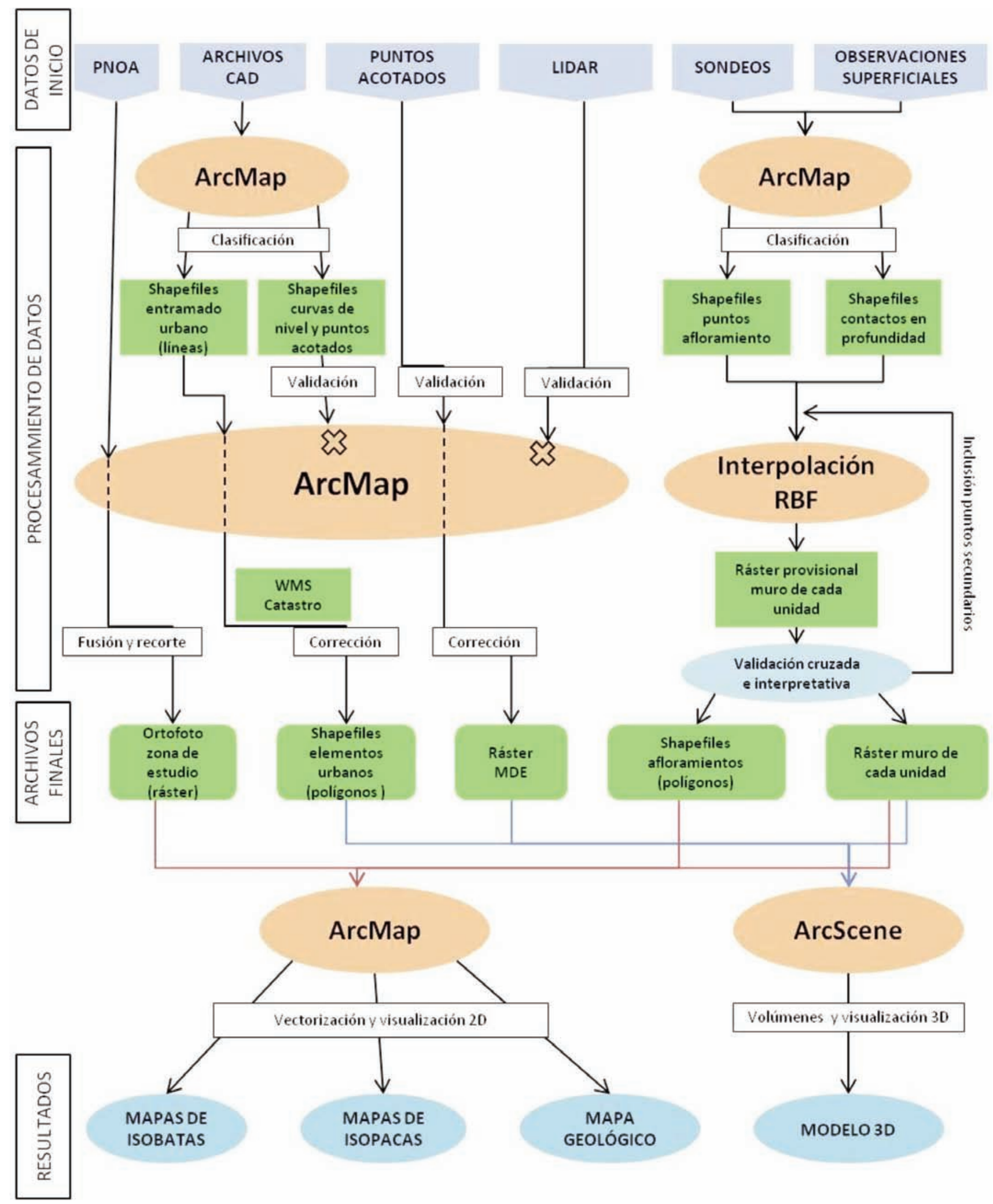

Figura 3. Diagrama de flujo que sintetiza la metodología seguida. Figure 3. Flowchart that summarizes the methodology followed. 


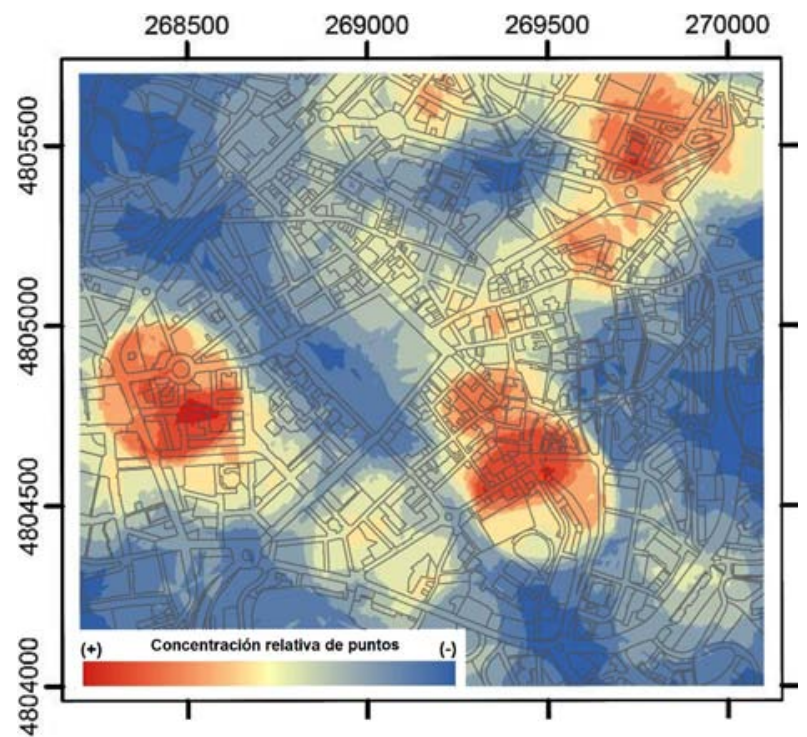

Figura 4. Distribución de puntos de reconocimiento utilizados para la reconstrucción del subsuelo.

Figure 4. Location of the site investigation points used for the reconstruction of the subsurface.

de 250 puntos por kilómetro cuadrado densidad que permite, con una distribución menos regular en los sondeos, cubrir toda la zona (Fig. 4). La localización concreta de las prospecciones no puede facilitarse por motivos de confidencialidad.

Ante las variaciones topográficas que experimentan las ciudades a causa de los movimientos de tierras, a modo de control de calidad, se compararon y corrigieron las desviaciones de la cota asignada en origen a los puntos de reconocimiento frente al MDE. Posteriormente se calculó la posición 3D de todos los contactos entre unidades en cada prospección. Los niveles seleccionados como objeto de la modelización fueron: formaciones superficiales (suma de suelo natural y rellenos antrópicos); Paleógeno sin distinción de tramos; y las tres formaciones cretácicas. La modelización fue planificada para caracterizar todos los contactos entre unidades, reflejados como superficies generadas mediante un interpolador a partir de los puntos de contacto en profundidad. Dada la distribución de prospecciones en agrupaciones (Fig. 4), se efectuó de forma preliminar un análisis exploratorio en busca de tendencias. Tras diversas pruebas, se interpoló mediante funciones de base radial, un método determinista exacto de carácter local que predice valores por encima de un máximo o debajo de un mínimo muestral, generando superficies suavizadas. En algunas zonas fue imprescindible aportar puntos secundarios para evitar anomalías como espesores inadmisibles o incompatibilidad con sondeos no usados en la interpolación. Una complicación añadida es que, pese a que las unidades cretácicas son concordantes, la falta ocasional de la Fm. Oviedo dificulta reconstruir su geometría.

La adecuación de las capas ráster se evaluó cuantitativa y cualitativamente. La primera comprobación supuso emplear validación cruzada, método que utiliza la totalidad de datos conocidos para estimar el modelo de autocorrelación del conjunto, omite un valor de cada vez y lo predice con el resto de muestras cotejando el resultado con el valor real; este proceso se repite en secuencia para cada punto. El parámetro matemático de referencia fue el error cuadrático medio. Este procedimiento fue utilizado asimismo para ajustar el interpolador. De forma paralela, cada superficie fue interpretada en términos de coherencia con el contexto geológico local (p. ej., buzamientos y orientaciones compatibles o espesores por encima del rango conocido). Finalmente, las superficies generadas se corrigieron y recortaron para asegurar consistencia espacial de superposición sin intersecciones inverosímiles.

El análisis de potenciales fracturas con componente vertical se desarrolló analizando las tendencias del contacto entre las Fms. Oviedo y La Argañosa. La búsqueda de variaciones de su cota impropias del rango de buzamientos habitual en unidades cretácicas fue afrontada estudiando las pendientes de dicha superficie. Este criterio evita incurrir en el error de vincular engrosamientos de la serie paleógena con el salto de fallas. La cartografía geológica de superficie se elaboró integrando los datos puntuales (primera unidad identificada en sondeos y observaciones in situ), las intersecciones entre el MDE y las capas ráster de contactos, y las fallas deducidas. La disposición y geometría de cada una de las unidades, sirviéndose de los contactos entre ellas, han sido representados en 2D mediante mapas de isolíneas obtenidas por vectorización de capas ráster clasificadas; es decir isobatas para las superficies de contacto e isopacas para los espesores tras efectuar operaciones algebraicas entre ellas.

Para la visualización del modelo geológico 3D, en el visor ArcScene, la ubicación espacial de las capas ráster que constituyen las superficies de muro y techo de las unidades viene fijada por su georreferenciado ( $x, y)$ y cota (z) implícita. La adaptación del soporte geográfico al entorno tridimensional supuso, en primer lugar, crear polígonos a partir de las cartografías topográficas que representan diferentes elementos del entramado urbano en superficie. Esto se realizó hasta un grado de detalle que se consideró suficiente para la visualización; en concreto el viario, las edificaciones en manzana cerrada y bloques aislados -con 


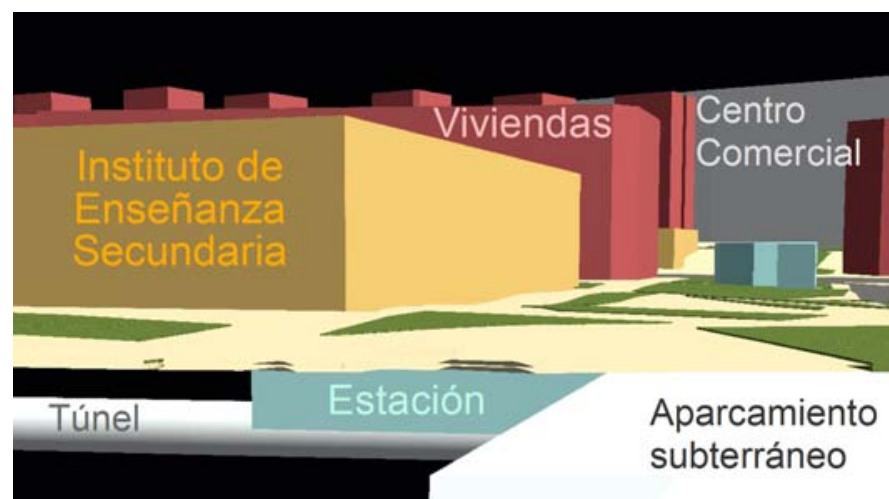

Figura 5. Recreación 3D de edificios e infraestructuras del entorno urbano.

Figure 5. 3D modelling of buildings and facilities of the urban environment.

diferenciación de usos para facilitar la identificación de inmuebles singulares-, las zonas verdes y algunos otros elementos (p. ej., fuentes). En el espacio subterráneo se incluyeron sótanos, aparcamientos subterráneos de hasta 5 plantas bajo rasante, así como los tubos correspondientes al trazado ferroviario de las dos líneas que atraviesan el centro urbano a profundidades que llegan a superar los $18 \mathrm{~m}$ (Fig. 5). Toda esta información fue ubicada en cota conforme al $\mathrm{MDE}$, y las volumetrías se simularon con extrusiones de los polígonos. Se incorporó una rejilla de coordenadas UTM como referencia espacial para enriquecer el modelo.

\section{Resultados y discusión}

En relación al marco altitudinal de partida surgieron diversos inconvenientes. EI MDE generado a partir de la cartografía topográfica autonómica fue desestimado por no proporcionar suficiente resolución vertical al contrastarlo con las cotas de emboquille de las prospecciones. Por su parte, dada la ingente cantidad de información contenida, resultó inviable filtrar la nube de puntos LIDAR para aislar la información topográfica del conjunto de datos correspondientes a elementos del entramado urbano. Fue necesario abrir una tercera vía para preparar el Modelo Digital de Elevaciones a utilizar en esta investigación. Tras extraer los puntos acotados de una cartografía municipal a escala 1:1.000 elaborada por el Consistorio en 1997, se corrigieron las áreas desactualizadas con datos de procedencia LIDAR. Esto precisó una inspección previa de los desajustes entre ambas referencias topográficas, recurriendo a puntos de control de cota invariante durante el lapso de tiempo que

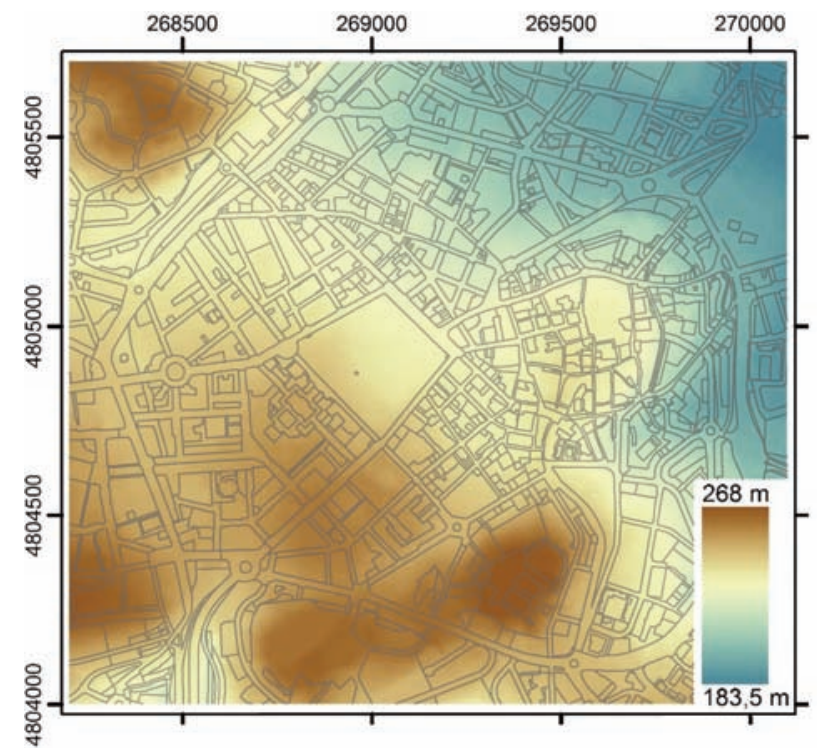

Figura 6. Modelo Digital de Elevaciones generado como soporte altitudinal del modelo 3D.

Figure 6. Digital Elevation Model that provides topographic reference for the $3 D$ model.

separa ambas fuentes de datos. Con una resolución de celdilla de $2 \mathrm{~m}$, el MDE refleja para la zona de estudio cotas mínima y máxima de 183,5 y 268 msnm (Fig. 6), y un relieve con pendientes comprendidas entre $0^{\circ}$ y $40^{\circ}$, siendo el promedio de $4^{\circ}$. Además de una mayor resolución espacial, respecto al MDE autonómico aporta las ganancias o pérdidas de cota más recientes experimentadas por el relieve de la ciudad.

La preparación del mapa geológico atendiendo al criterio de unidad más superficial descrita en los puntos de reconocimiento no es inmediata, ante los movimientos de tierras acontecidos en los últimos años. Fue necesario comparar la cota original de cada prospección con la del MDE en su localización, para identificar en la testificación la unidad actualmente más somera. En el mapa que se presenta se han eliminado las formaciones superficiales para no enmascarar el sustrato natural (Fig. 7). El $72 \%$ de la superficie estudiada está ocupada por afloramientos de materiales paleógenos, y el $28 \%$ restante por cretácicos. Entre estos últimos, un $26 \%$ corresponde a la $\mathrm{Fm}$. Oviedo mientras que la Fm. La Argañosa queda restringida a retazos. No hay evidencias de que llegue a aflorar la Fm. San Lázaro. Con respecto a mapas geológicos previos (Gutiérrez Claverol and Torres Alonso, 1995; González Fernández et al., 2004) los afloramientos cretácicos se reducen, especialmente en el caso de la Fm. La Argañosa, mientras que los contactos entre unidades cretácicas y materiales paleógenos son más alabeados. 


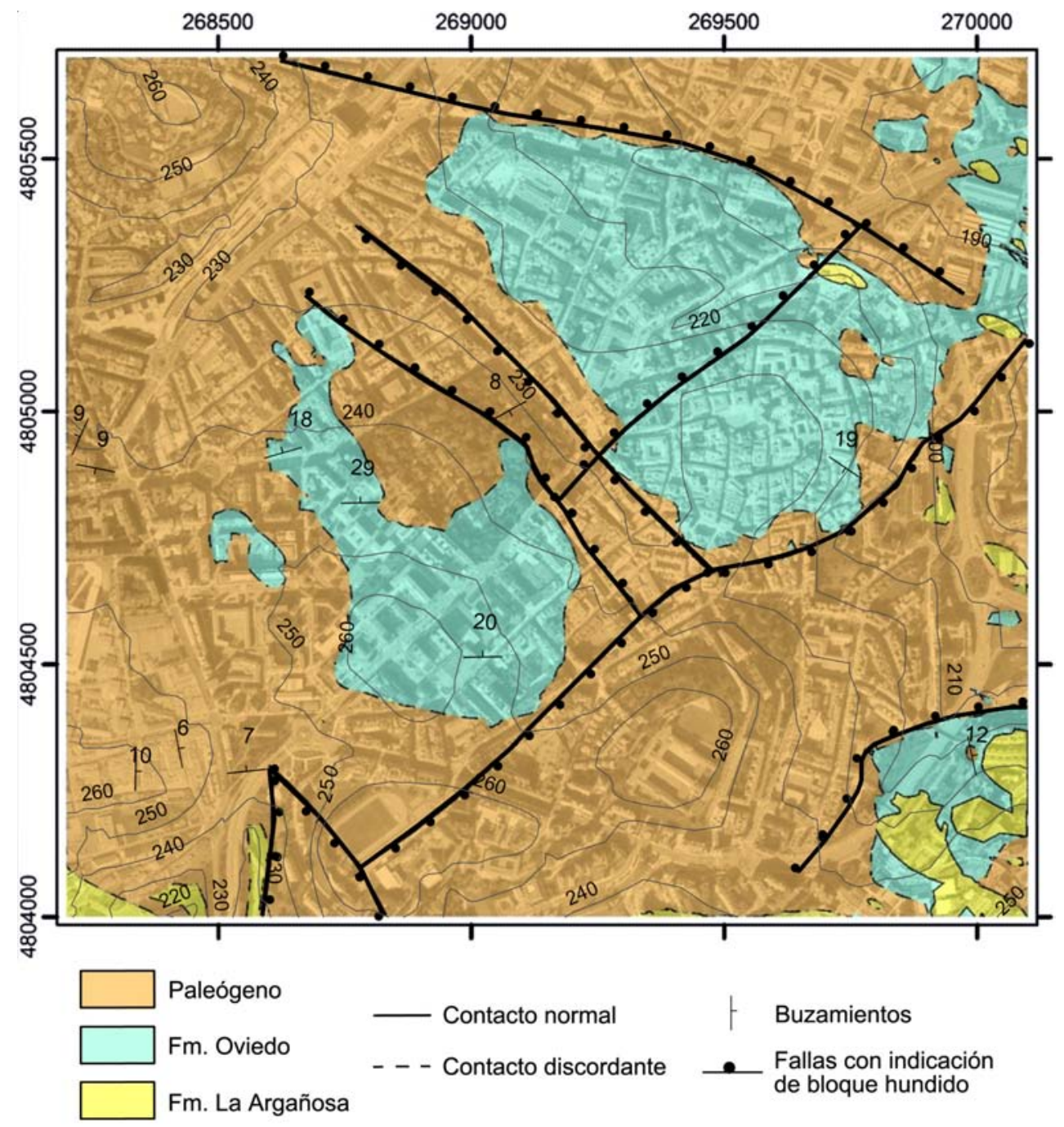

Figura 7. Cartografía geológica actualizada del centro de Oviedo. Figure 7. Updated geological map of the urban centre of Oviedo.

La cartografía geológica muestra asimismo las potenciales fallas más importantes que afectan a la cobertera meso-paleógena. Éstas se han deducido por representar inflexiones abruptas en el contacto interpolado entre la Fm. Oviedo y Fm. La Argañosa (Fig. 8). Se trata de fracturas de trazado moderadamente rectilíneo cuyas orientaciones NE-SO y NO-SE resultan coherentes con los sistemas conocidos en la cuenca (Gutiérrez Claverol and Torres Alonso, 1995), atribuyéndoseles saltos verticales superiores a 10 metros. No se puede precisar hacia dónde se inclinan las superficies de falla y con ello el funcionamiento normal o inverso de las mismas. No es objetivo de esta investigación discutir su origen y cinemática, pero la principal fractura cartografiada, situada en el margen SE y rumbo NE-SO, podría responder a la reactivación por deslizamiento flexural de cabalgamientos variscos plegados, según el modelo de Pulgar et al. (1999). La segunda fractura interpretada más destacada en el mapa, la más septentrional y de dirección ONO-ESE, se corresponde con una falla recogida por la cartografía continua del IGME, mostrando descenso del bloque norte.

Por su envergadura estas fallas pueden controlar el patrón de afloramiento de las formaciones cretácicas, sin embargo, frente a la abundancia de contactos mecánicos interpretados por Gutiérrez Claverol and Torres (1995), en el mapa obtenido el paleorrelieve posee mayor relevancia como condicionante en la delimitación de los afloramientos. Dicho lo anterior, la observación en detalle de la superficie de techo de la Fm. Argañosa permite advertir otras anomalías loca- 


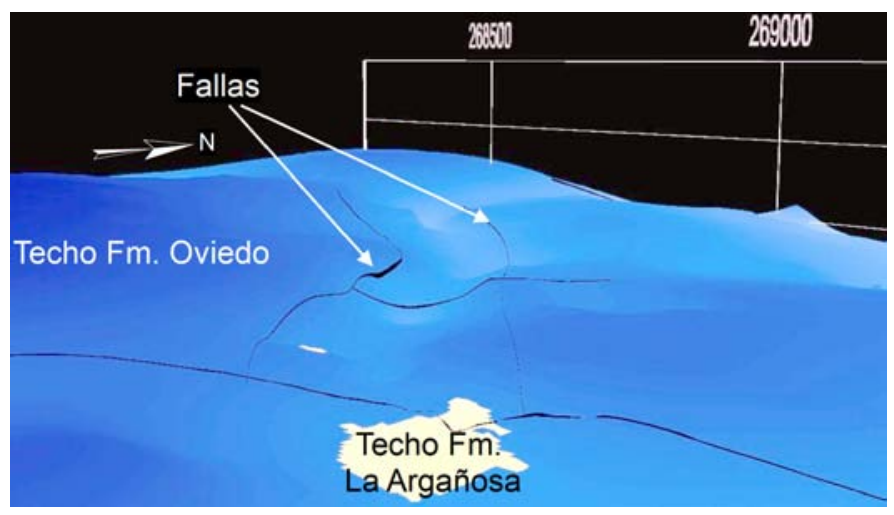

Figura 8. Ejemplo de potenciales fallas deducidas a partir de inflexiones en el contacto Fm. Oviedo-Fm. La Argañosa.

Figure 8. Example of potential faults deduced from inflections in the contact between Oviedo Fm and La Argañosa Fm.

les compatibles con la presencia de fracturas de menor desplazamiento y continuidad lateral, no cartografiables a esta escala de trabajo, de cuya presencia da cuenta el mapa geológico de Martínez Álvarez and Torres Alonso (1968).

En relación a la geometría de las unidades del subsuelo, se han realizado una serie de mapas de isobatas e isopacas. Para la confección de los mismos se han tenido en cuenta las formaciones superficiales, no representadas en la Fig. 7. Del análisis de los mapas de isobatas e isopacas generados pueden realizarse las siguientes observaciones:

- Formaciones superficiales. Toda la zona de estudio está cubierta por, al menos, unos decímetros de recubrimiento con una disposición en planta y profundidad muy irregular. En zonas en que es conocida la existencia de importantes rellenos antrópicos se han estimado valores que superan los $10 \mathrm{~m}$, con máximos puntuales en torno a $18 \mathrm{~m}$. En los casos de rellenos por sedimentos aluviales, las potencias pueden Ilegar a alcanzar los $9 \mathrm{~m}$. Para ilustrar la distribución de estos materiales se muestran los espesores superiores a $3 \mathrm{~m}$ (Fig. 9a). Este valor posee relevancia a efectos de planificación de estudios geotécnicos según establece el
Documento Básico Seguridad EstructuralCimientos del Código Técnico de la Edificación (Ministerio de Vivienda, 2006), pues supone cambiar la categoría de los terrenos y con ello la intensidad de reconocimiento exigible a las campañas de prospección.

- Depósitos paleógenos. El techo de la unidad se encuentra hasta un máximo de $18 \mathrm{~m}$ de profundidad (Fig. 9b) mientras que su base, al representar un paleorrelieve, es muy irregular. Como resultado la potencia de estos depósitos es variable, siendo lo habitual que no supere los 15-20 m (Fig. 9c) si bien en el extremo SO se ha determinado un espesor máximo de hasta 40 $\mathrm{m}$. Cabe señalar que en algunos puntos de la mitad sur del centro urbano la Fm. Oviedo ha sido totalmente erosionada y los materiales paleógenos se apoyan directamente sobre las arenas de la Fm. La Argañosa. El conglomerado calcáreo situado a muro de la serie paleógena, desarrollado por el desmantelamiento de la Fm. Oviedo, muestra en dicha zona sus mayores potencias en la ciudad, llegando a exceder los $15 \mathrm{~m}$.

- Fm. Oviedo. La localización de su techo varía ampliamente, encontrándose a más de $35 \mathrm{~m}$ de profundidad en los márgenes NO y SE de la zona de estudio, lo que contrasta con el eje central de la cuenca, donde la unidad aflora o yace muy somera bajo la serie paleógena (Fig. 9d). $\mathrm{Su}$ espesor también resulta muy cambiante, aunque se mantiene por debajo de $15 \mathrm{~m}$ en la mayor parte del área investigada (Fig. 9e). Se ha estimado que podría alcanzar los $30 \mathrm{~m}$ de potencia en el extremo NO.

- Fm. La Argañosa. La información sobre la profundidad que alcanza esta unidad es limitada ya que solo tres sondeos cortan su muro, pero permite confirmar su espesor en la zona estudiada entre 35 y $40 \mathrm{~m}$. Por su parte, el techo muestra una tendencia global de descenso de cota hacia el NO, en el cuadrante noroccidental con pendiente variable entre 2 y $6^{\circ}$, alcanzando una profundidad máxima de $70 \mathrm{~m}$ (Fig. 9f). Cabe destacar que este contacto en la ciudad posee incidencia constructiva, ya que represen-

Figura 9. (a) Distribución de las formaciones superficiales cuya potencia supera los $3 \mathrm{~m}$; (b) Isobatas de techo de la serie paleógena; (c) Isopacas de la serie paleógena; (d) Isobatas de techo de la Fm. Oviedo; (e) Isopacas de la Fm. Oviedo; (f) Isobatas de techo de la Fm. La Argañosa.

Figure 9. (a) Distribution of superficial formations with thicknesses exceeding $3 \mathrm{~m}$; (b) Roof isobath map of the Paleogene deposits; (c) Isopach map of the Paleogene deposits; (d) roof isobaths map of the Oviedo Fm.; e) Isopach map of the Oviedo Fm.; f) roof isobath map of the La Argañosa Fm. 


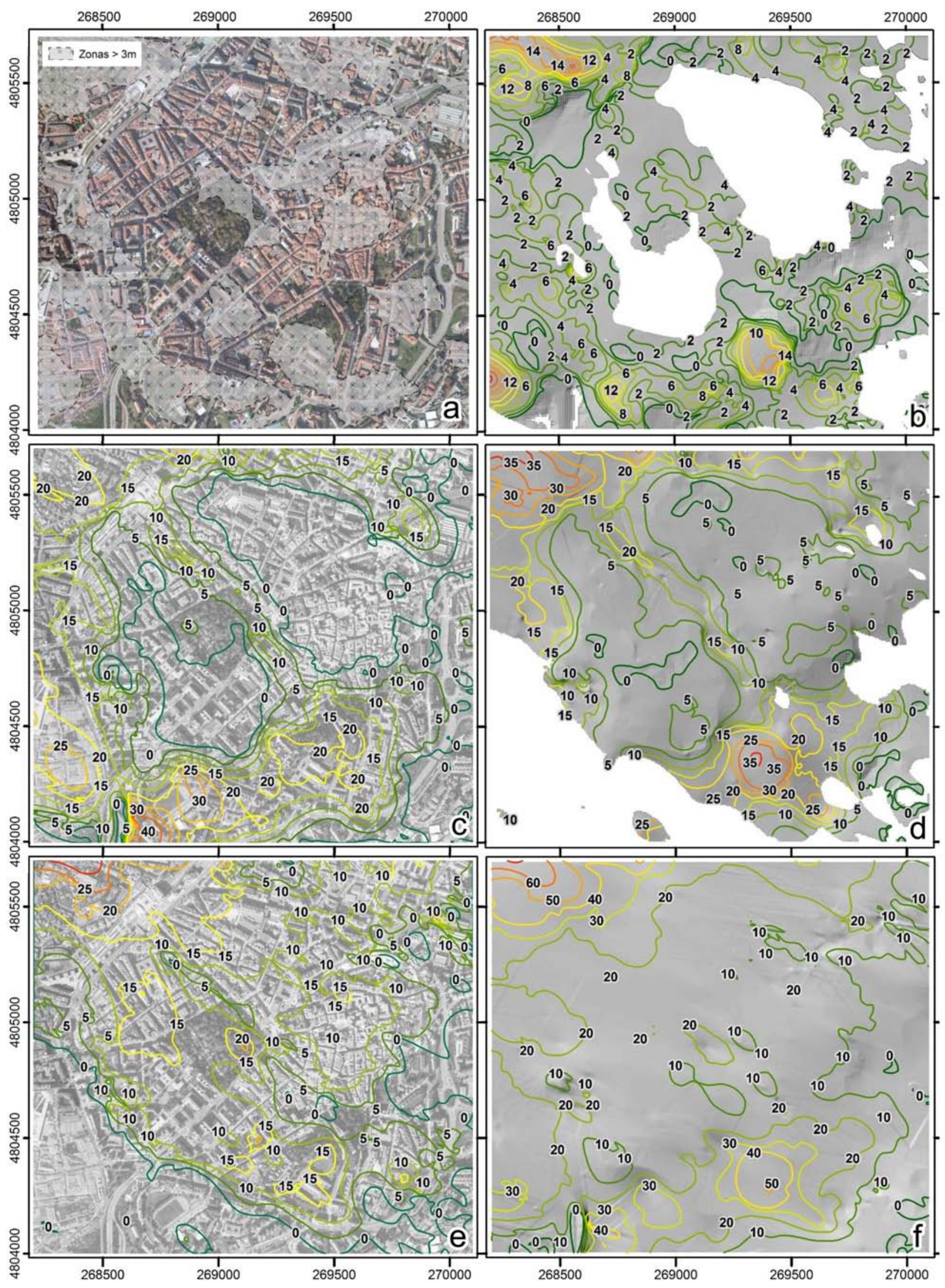


ta el tránsito en profundidad de una unidad rocosa a otra dominada por materiales que ofrecen condiciones de cimentación y estabilidad de excavaciones significativamente peores (Pando et al., 2012). Su presencia ha condicionado en numerosos proyectos el número de plantas subterráneas a ejecutar.
Todas las superficies ráster generadas que representan los contactos entre unidades geológicas, así como la reconstrucción de elementos superficiales y subterráneos del entramado urbano, fueron integrados en un modelo tridimensional conjunto basado en capas (Fig. 10a). El bloque 3D creado posee las dimensiones en planta del área de estudio, y alcanza
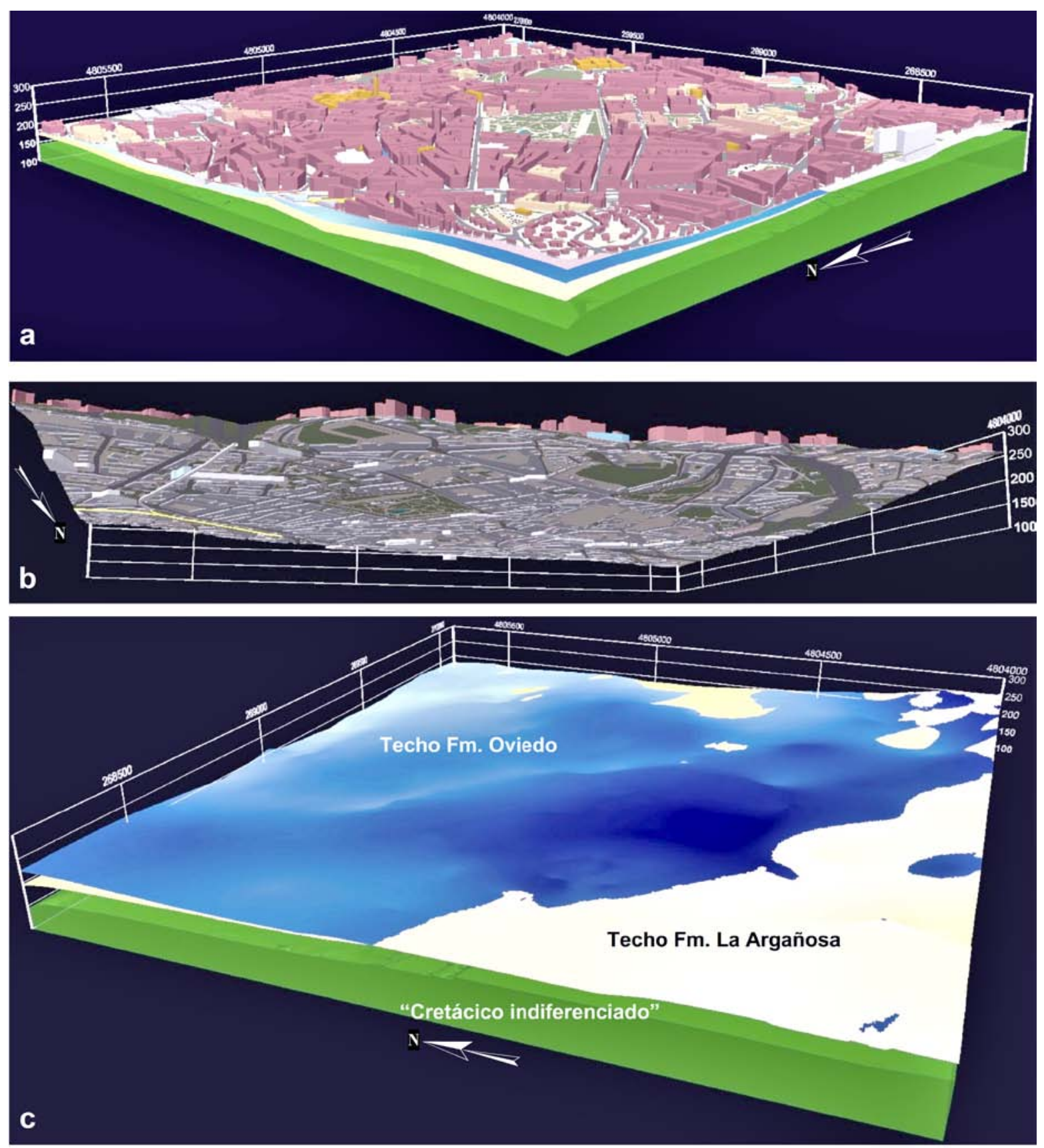

Figura 10. (a) Aspecto general de las capas que conforman el modelo 3D; (b) Vista subterránea de la capa urbana; (c) Apariencia del paleorrelieve, apreciándose la morfología irregular del techo de la Fm. Oviedo.

Figure 10. (a) General view of the layers that shape the 3D model; (b) Bottom view of the urban layer; (c) 3D view of the palaeorelief, where the irregular geometry of the Oviedo Fm. top can be appreciated. 
una profundidad máxima de $168 \mathrm{~m}$. Este valor está condicionado -aparte de por el relieve urbano- por los datos disponibles más profundos.

Esta representación verificó la consistencia altitudinal de todos los elementos y una interacción espacial coherente entre las capas, lo que se traduce en una superposición correcta y sin solapes de los contactos, e intersecciones de estos últimos con el MDE ajustadas a las trazas cartográficas que delimitan las unidades. Por otro lado, la adición de infraestructuras subterráneas dota al modelo de un significativo grado de realismo, al tiempo que mejora la percepción de escala al observar el medio geológico (Fig. 10b). Asimismo, rasgos como la geometría del paleorrelieve pueden observarse de una forma inédita hasta ahora (Fig. 10c), facilitando la identificación de las zonas en las que la Fm. Oviedo ha sido totalmente erosionada y ayudando a prevenir los problemas geotécnicos derivados de su ausencia. La base del modelo está representada por el "Cretácico Indiferenciado", que corresponde mayoritariamente a la $\mathrm{Fm}$. San Lázaro. En algunas zonas podría llegar a incluir formaciones subyacentes pero la ausencia de datos imposibilita discriminar los contactos estratigráficos.

\section{Conclusiones}

Esta investigación ha supuesto la primera aproximación a la configuración 3D de las unidades más someras que conforman el subsuelo del centro urbano de Oviedo, partiendo de información geológica procedente de sondeos mecánicos y observaciones in situ almacenados en una base de datos implementada en un SIG.

La experiencia demuestra que este tipo de reconstrucciones deben estar apoyadas en un soporte topográfico actualizado y con la suficiente densidad de datos, requiriendo en este caso la construcción de un MDE específico con resolución espacial de $2 \mathrm{~m}$. El mapa geológico obtenido muestra mayor proporción de afloramientos paleógenos que las cartografías anteriores, aportando contactos más condicionados por el paleorrelieve excavado en materiales cretácicos que por parte de fracturas. Incluye asimismo varias fallas novedosas, mientras que otras se confirman respecto a mapas previos. Todas ellas con trazados compatibles con las orientaciones regionales.

La interpolación 3D de los contactos estratigráficos por medio de funciones de base radial, permitió generar superficies óptimas en términos de error de predicción y coherencia geológica. Las formaciones superficiales se distribuyen de forma irregular por toda la zona de estudio con un espesor máximo de 18 $\mathrm{m}$, mientras que los materiales paleógenos subyacentes ofrecen potencias normalmente inferiores a 20 $\mathrm{m}$. La Fm. Oviedo, cuyo espesor máximo se cifra en 10-15 m en la mayor parte del centro urbano, llega a desaparecer a causa del marcado paleorrelieve presente, especialmente, en el extremo S-SO. Por su parte, la Fm. La Argañosa presenta un espesor coherente con los datos disponibles en otros puntos de la cuenca, estando su techo localizado a una profundidad máxima de $70 \mathrm{~m}$ y con tendencia general buzante hacia el NO.

La experiencia desarrollada ha demostrado para la zona de estudio un aprovechamiento efectivo del contenido de estos repositorios digitales, ratificando la importancia de impulsar su creación en ciudades de tamaño medio a pequeño, y no solo en grandes áreas urbanas, como es tendencia habitual. Los resultados ofrecidos por el modelo basado en capas servirán como referencia preliminar para futuros modelos 3D basados en la discretización del espacio subterráneo en unidades volumétricas, lo que permitirá ampliar el alcance de las investigaciones al estudio de otras variables del terreno.

La integración del espacio subterráneo con una virtualización del entramado urbano en superficie, proporciona un modelo visualmente efectista que ofrece una gran versatilidad para la interpretación y difusión de resultados.

\section{Agradecimientos}

Los autores muestran su gratitud a todas las empresas y profesionales que han cedido desinteresadamente los datos geológicos utilizados, sin los cuales este tipo de investigaciones resultan inabordables. Se hace extensivo el agradecimiento al Dr. Luis Quintana, así como a otro revisor anónimo, cuyas labores han permitido mejorar sin duda la calidad del artículo.

\section{Referencias}

Alonso, J.L., Pulgar, J., García-Ramos, J.C. and Barba, P. 1996. Tertiary basins and alpine tectonics in the cantabrian mountains (NW Spain). In: Friend, P.F. and Dabrio, C. (eds.), Tertiary Basins of Spain. Tectonics, climate and sea-level changes. Cambridge University Press, Cambridge, 214-227.

Campbell, S.D.G., Merritt, J.E., Dochartaigh, B.E.O., Mansour, M., Hughes, A.G., Fordyce, F.M., Entwisle, D.C., Monaghan, A.A. and Loughlin, S.C. 2010. 3D geological models and their hydrogeological applications: support- 
ing urban development - a case study in Glasgow-Clyde, UK. Zeitschrift der Deutschen Gesellschaft fur Geowissenschaften, 161 (2), 251-262.

Campbell, D., De Beer, J., Lawrence, D., Van der Meulen, M., Mielby, S., Hay, D., Scanlon, R., Van Campenhout, I., Taugs, R. and Eriksson, I. 2014. Sub-Urban. Transforming relationships between geoscientists and urban decisionmakers (COST Action TU1206). 14th World Conference of Associated Research Centers for the Urban Underground Space, Seoul, 284-289.

Devleeschouwer, X. and Pouriel, F. 2009. Brussels Urban Geology (BUG): a 2D and 3D model of the underground by means of GIS. In: Culshaw, M.G., Reeves, H.J., Jefferson, I. and Spink, T.W. (eds.), Engineering Geology for Tomorrow's Cities, Engineering Geology Special Publication 22. Geological Society, London, paper 420.

De Beer, J., Price, S.J. and Ford, J.R. 2012. 3D modelling of geological and anthropogenic deposits at the World Heritage Site of Bryggen in Bergen, Norway. Quaternary International, 251, 107-116.

Díaz-Díaz, L.M. 2016. Caracterización geológica y geotécnica del subsuelo urbano de Avilés y su entorno. Tesis Doctoral, Universidad de Oviedo, 314 págs.

Eriksson, I., Borchgrevink, J., Saether, S.S., Daviknes, H.K., Adamou, S. and Adresen, L. 2016. Oslo. TU1206 COST Sub-Urban WG1 Report, 36 págs.

García-Ramos, J.C. and Gutiérrez Claverol, M. 1995. La cobertera mesozoico-terciaria. In: Aramburu, C., Bastida, F. (eds.), Geología de Asturias. Trea, Gijón, 81-94.

GEODE. Mapa Geológico Digital continuo de España [en línea]. [Fecha de consulta 20/10/2017]. Disponible en: http://mapas.igme.es/gis/services/Cartografia_Geologica /IGME_Geode_50/MapServer/WMSServer

González Fernández, B., Gutiérrez Claverol, M. y Menéndez Casares, E. 2005. Caracterización hidrogeológica de la sucesión cretácica en el sector central de Asturias (Oviedo, NO de España). Boletín Geológico y Minero, 116 (3), 231-245.

González Fernández, B., Menéndez Casares, E., Gutiérrez Claverol, M. y García-Ramos, J.C. 2004. Litoestratigrafía del sector occidental de la cuenca cretácica de Asturias. Trabajos de Geología, Universidad de Oviedo, 24, 43-80.

González-Fernández, B., Menéndez-Casares, E., MeléndezAsensio, M. and Gutiérrez-Claverol, M. 2010. Mining activity and geotechnical and hydrogeological issues related to the presence of gypsum in Asturias (NW Spain). In: Sampson, D.H. (ed.), Gypsum: properties production and applications. Nova Publishers, USA, 35-68.

Gutiérrez Claverol, M. and Torres Alonso, M. 1995. Geología de Oviedo. Descripción, recursos y aplicaciones. Paraíso, Oviedo, 276 pp.

Gutiérrez Claverol, M., Pando, L. y García-Ramos, J.C. 2008. Procesos y productos de alteración de formaciones rocosas en Asturias y su repercusión socio-económica. Boletín Geológico y Minero, 119 (2), 211-230.

Gutiérrez Claverol, M., Torres Alonso, M. y Luque Cabal, C. 2002. El subsuelo de Gijón: Aspectos Geológicos. CQ Licer, S.L., Oviedo, 462 pp.
Hoyer, A.S., Jorgensen, F., Sandersen, P.B.E., Viezzoli, A. and Moller, I. 2015. 3D geological modelling of a complex buried-valley network delineated from borehole and AEM data. Journal of Applied Geophysics, 122, 94-102.

Julivert, M., Fontboté, J. M., Ribeiro, A. y Conde, L. 1972. Mapa tectónico de la Península Ibérica y Baleares. Instituto Geológico y Minero de España, Madrid, 113 pp.

Martínez Álvarez, J.A. and Torres Alonso, M. 1968. Esquema geológico y geotécnico de la zona urbana de la ciudad de Oviedo. ETS de Ingenieros de Minas de Oviedo, 1 pág.

Merritt, J., Entwisle, D. and Monaghan, A. 2009. Integrated geoscience data, maps and 3D models for the City of Glasgow, UK. In: Culshaw, M.G., Reeves, H.J., Jefferson, I. and Spink, T.W. (eds.), Engineering Geology for Tomorrow's Cities, Engineering Geology Special Publication 22. Geological Society, London, paper 394.

Mielby, S. and Sandersen, P.B.E. 2017. Development of a 3D geological/hydrogeological model targeted at sustainable management of the urban water cycle in Odense City, Denmark. Procedia Engineering, 209, 75-82.

Ministerio de Vivienda (2006): Código Técnico de la Edificación (CTE). Real Decreto 314/2006 de 17 de marzo. Publicado en BOE de 28 de marzo de 2006.

Pando, L., Díaz-Díaz, L.M. and López-Fernández, C. 2018. Urban geology and geotechnics by using GIS-based databases: the case of Asturian cities (NW Spain). 2nd International Research Conference on Sustainable Energy, Engineering, Materials and Environment, Mieres, 46-47.

Pando, L., Gutiérrez Claverol, M. y Flor-Blanco, G. 2012. Propiedades geotécnicas de la Formación La Argañosa (Cretácico Superior, Oviedo). Boletín Geológico y Minero, 123 (1), 65-75.

Pando, L., Pulgar, J.A. and Gutiérrez-Claverol, M. 2013. A case of man-induced ground subsidence and building settlement related to karstified gypsum (Oviedo, NW Spain). Environmental Earth Sciences, 68 (2), 507-519.

Pando, L., Díaz-Díaz, L.M., Arias, D. and Flor-Blanco, G. 2014. A case study of GIS-based geotechnical database in urban environment (Oviedo, NW Spain). European Geosciences Union General Assembly, Vienna, 16, EGU2014-7518-1.

Pando, L., Martínez-Largo, J.A., Flor-Blanco, G. y Díaz-Díaz, L.M. 2016. Base de datos geotécnicos de Gijón: desarrollo y aplicaciones. IX Congreso Geológico de España, Huelva, Geo-Temas 16, 973-976.

Pulgar, J.A., Alonso, J.L., Espina, R.G. y Marín, J.A. 1999. La deformación alpina en el basamento varisco de la Zona Cantábrica. Trabajos de Geología, 21, 283-294.

Royse, K.R., Rutter, H.K. and Entwisle, D.C. 2009. Property attribution of 3D geological models in the Thames Gateway, London: new ways of visualising geoscientific information. Bulletin of Engineering Geology and the Environment, 68, 1-16.

Royse, K.R., Bricker, S.H., Jackson, C.R., Kingdon, A. and Hughes, A.G. 2015. The development of linked databases and environmental modelling systems for decision- 
making in London. In: Lollino, G., Manconi, A., Culshaw, M., Bobrowsky, P. and Luino, F. (eds), Engineering geology for society and territory, 5, 1195-1199. Stafleu, J., Maljers, D., Gunnik, J.L., Menkovic, A. and Busschers, F.S. 2011. 3D modelling of the shallow subsurface of Zeeland, the Netherlands. Netherlands Journal of Geosciences - Geologie en Mijnbouw, 90 (4), 293-310.

Tonini, A., Guastaldi, E. and Meccheri, M. 2009. Three- dimensional reconstruction of the Carrara Syncline (Apuane Alps, Italy): An approach to reconstruct and control a geological model using only field survey data. Computers \& Geosciences, 35, 33-48.

Veeger, A.I., Murray, D.P., Hermes, O.D., Boothroyd, J.C. and Hamidzada, N.A. 2004. Harnessing the power of relational databases for managing subsurface geotechnical and geologic data. Environmental and Engineering Geoscience, 10 (4), 339-346.

Recibido: septiembre 2018

Revisado: febrero 2019

Aceptado: abril 2019

Publicado: septiembre 2019 
